Approach to the diagnosis of neurotransmitter diseases exemplified by the differential diagnosis of childhood-onset dystonia. Ann Neurol 2003;54(suppl 6):S18-S24). (Respond: Dr Assmann, University Children's Hospital, Moorenstrasse 5, 40225 Dusseldorf, Germany).

COMMENT. The term dystonia is used for a symptom or abnormal movement disorder. Dystonia is characterized by intermittent or continuous muscle spasms that are generalized (dystonia musculorum deformans) or segmental (localized to the neck (spasmodic torticollis), upper limb, or lower limb), and primary genetic or acquired (postperinatal asphyxia, trauma, toxins, or vascular). In primary hereditary dystonias, the mode of transmission is variable. One form with high incidence in Ashkenhazi Jews is probably autosomal dominant. The first symptom in hereditary dystonia is an involuntary posturing of one portion of the body, most commonly a plantar, flexion-inversion movement of the foot, commonly misdiagnosed as a hysterical gait. In treatment, trihexyphenidyl (Artane) is used in Ashkenazi Jewish patients, and Levodopa, alone or with a decarboxylase inhibitor (Sinemet), in patients with late onset dystonia. Cryothalamectomy is a surgical approach rarely entertained in children with medically-intractable hemidystonia.

CSF analysis and magnetic resonance spectroscopy in the diagnosis of neurotransmitter diseases are reviewed by Hyland K (Ann Neurol 2003;54(suppl 6):S13S17), and Novotny EJ Jr et al (Ann Neurol 2003;54(suppl 6):S25-S31). The CSF compounds measured are homovanillic acid (end product for dopamine metabolism), 5hydroxyindoleacetic acid (for serotonin), and 3-methoxy-4-hydroxyphenylglycol (for norepinephrine). MRS methods are under investigation for the measurement of neurotransmitters in the brain. Abnormalities of motor cortex excitability preceding voluntary movement in patients with dystonia have been studied by Gilio $\mathrm{F}$ et al (Brain 2003;126:1745-1754). Dystonic movements are commonly triggered or made worse by voluntary action.

\title{
PAROXYSMAL DYSKINESIAS
}

Clinical characteristics of 26 children diagnosed with paroxysmal dyskinesias between 1980 and 2000 were evaluated retrospectively at the National Neurological Institute "C Besta" of Milan, Italy. Patients were categorized according to precipitating factors: 14 had paroxysmal kinesigenic dyskinesia (PKD), 6 had paroxysmal nonkinesigenic dyskinesia (PNKD), and 6 had paroxysmal exercise-induced dyskinesia (PED). None had paroxysmal hypnogenic dyskinesia (PHD), a form of nocturnal frontal lobe epilepsy. Patients with PKD had a mean age at onset of 7.1 years (range1.5-14 years); 13 were idiopathic, with a positive family history in 9 and autosomal-dominant inheritance; and one was associated with Chiari type 1 malformation. Of the 6 with PNKD, 1 had multiple sclerosis, 2 had cerebral palsy, 1 had a left basal ganglia stroke. 1 an acute inflammatory encephalopathy, and only 1 was idiopathic. Six with PED were all idiopathic, and attacks of dystonia or choreoathetosis were triggered by prolonged exercise, usually running or walking. Antiepileptic drugs, especially carbamazepine, were most effective in treatment of the PKD type, with benefit obtained in $70 \%$. The occasional co-occurrence of epilepsy and PKD may be explained by a common ion channel 
dysfunction, and a gene locus mapped to chromosome 16. (Zorzi G, Conti C, Erba A et al. Paroxysmal dyskinesias in childhood. Pediatr Neurol March 2003;28:168-172). (Respond: Giovanna Zorzi MD, Department of Child Neurology, Instituto Nazionale Neurologico "C Besta", Milano, Italy).

COMMENT. Paroxysmal dyskinesias refer to brief attacks of dystonia or choreoathetosis, alone or combined, with return to normal between episodes. Cases are classified according to precipitating factors. Paroxysmal kinesthetic dyskinesia (PKD) is the most frequent form of paroxysmal dyskinesia, it is usually familial and idiopathic, often responds to treatment with carbamazepine, and may resolve spontaneously by age 30. PNKD is relatively rare, it is often symptomatic of illnesses including cerebral palsy, infection, or vascular lesion, and response to treatment is poor. Exercise-induced (PED) cases are rare, usually in response to running, and dystonia is generalized or focal, affecting the foot. Antiepileptic drugs, including carbamazepine, clonazepam, and acetazolamide, L-Dopa, and trihexyphenidyl are reported of benefit in some cases.

\section{LANGUAGE DISORDERS}

\section{LANGUAGE REGRESSION, AUTISM, AND EPILEPSY}

The records of 196 consecutive children (143 males and 53 females) with language regression evaluated between 1988 and 1994 by a child neurologist were analyzed at the Department of Neurology, Albert Einstein College of Medicine, Bronx, NY. Patients with a neurodegenerative or metabolic disorder, acquired brain injury, or Rett syndrome were excluded. Mean age at regression, defined as loss of previously acquired language skills, was 21.2 months (SD 10.5), the mean age at first visit was 50.6 months (SD 41.1), and the mean interval between onset and referral was delayed for 34.8 months (SD 38.3). Trigger factors that preceded the onset of language regression were reported in $74(38 \%)$; these were definite in $34(17 \%)$ and possibly related in $40(20 \%)$. They included illness $(23 \%)$, emotional upset $(16 \%)$, seizures $(3 \%)$, and trauma (1\%). A history of seizures was present in $15 \%$, but seizures were temporally related to language regression in only $3 \%$. Repeated ear infections were the most frequently reported abnormality in the past history, occurring in $32 \%$. At the time of neurologic examination, autistic spectrum disorder was diagnosed in $93 \%$, and cognitive impairment occurred in $73 \%$, with severe mental retardation in $3 \%$. Hearing was normal in $94 \%$, and mildly impaired in $6 \%$. Stereotypies were noted in $85 \%$, hypotonia in $58 \%$, toe walking in $15 \%$, and oromotor deficits in $11 \%$. Sensorimotor development was normal in $29 \%$. CT/MRI obtained in 75 children was abnormal in only 3 (4\%). Chromosomes and metabolic tests were normal in all 47 tested. EEGs showed paroxysmal abnormalities in 4 of $12(33 \%)$ with prolonged video monitoring. At follow-up (mean age 64 months) 39\% were nonverbal and an additional 45\% showed decreased verbal output. Some recovery occurred in $61 \%$ but only one child recovered fully. Improvement was most likely in the $49 \%$ who were developmentally normal before language regression was noted. (Wilson S, Djukic A, Shinnar S et al. Clinical characteristics of language regression in children. Dev Med Child Neurol August 2003;45:508-514). (Respond: Isabelle Rapin MD, Albert Einstein College of Medicine, K807, 1300 Morris Park Ave, Bronx, NY 10461). 